\title{
Soft PLC Research And Development System Based On PC
}

\author{
ZHOU Wenjun ${ }^{1, a}$ \\ ${ }^{1}$ Nanning College for Vocational Technology, Nanning, 530008, Guangxi, China \\ aZhouWenjun1031@yeah.net
}

Keywords: Soft PLC Research; PC; Development

\begin{abstract}
With the development of industrial control technology, the drawbacks of traditional PLC gradually emerged. Although most of the PLC manufacturers have similar programming language, the PLC hardware and software products are not compatible. Also, their configuration, addressing and grammatical structure are inconsistent. Every PLC company uses their own program software which is quite expensive, less compatible and lots are needed to operate under DOS environment. This leads to inconvenience to users and also is not conducive to the PLC program transplant. With the development of control technology, the inconsistency has become increasingly prominent. PLC technology is a newly developed PC-based technology, which is in accordance with IEC61131-3 international programming standards and having good openness and cost-effective. Actually soft and traditional PLC operate base on same theory in terms of their functions, it can be divided into development system and operation system. Through the development of PLC editing system, it can be downloaded into the operating system for the control functions.
\end{abstract}

\section{Advantages of Soft PLC technology and its system}

\section{Advantages of Soft PLC}

A. Open system architecture. Soft PLC has a wide range of I / 0 ports and a variety of field bus interface, which supports a variety of hardware. Users can choose the appropriate hardware based on their own requirements for the composition of soft PLC.

B. Sufficient instruction and control module. The instructions of traditional PLC are fixed; however, the actual industrial control may need to further define their algorithms. Soft PLC supports a variety of control algorithms and also users can embed their own control algorithms.

C. Enhanced data processing capabilities. Make full use of the PC resources, for example, greater capacity memory, high-speed CPU and other hardware.

D. Strong network communication. Soft PLC not only connects to enterprise management information system, facilitating the integration of enterprises, but also monitors industrial facility operation, achieving data transmission and online monitoring, editing, simulation, debugging and other functions.

E. Standardized editing language. Soft PLC technology is developed based on the IEC61131-3 standard, thus, it would be easier to master the standard language development.

Although soft PLC has various advantages, this technique still has some problems needed to be solved. For instance, the reliability of the equipment that required to work in a worse industrial environment; the real-time control, the preferred operating system of soft PLC is Windows NT, however, it is not a hard real-time operating system. Traditional PLC is a hard real-time, and so, it can provide fast, deterministic and repeatable reaction.

\section{Soft PLC system architecture}

Comparison between soft and traditional PLC:

The construction of traditional PLC is shown as the figure 1. 


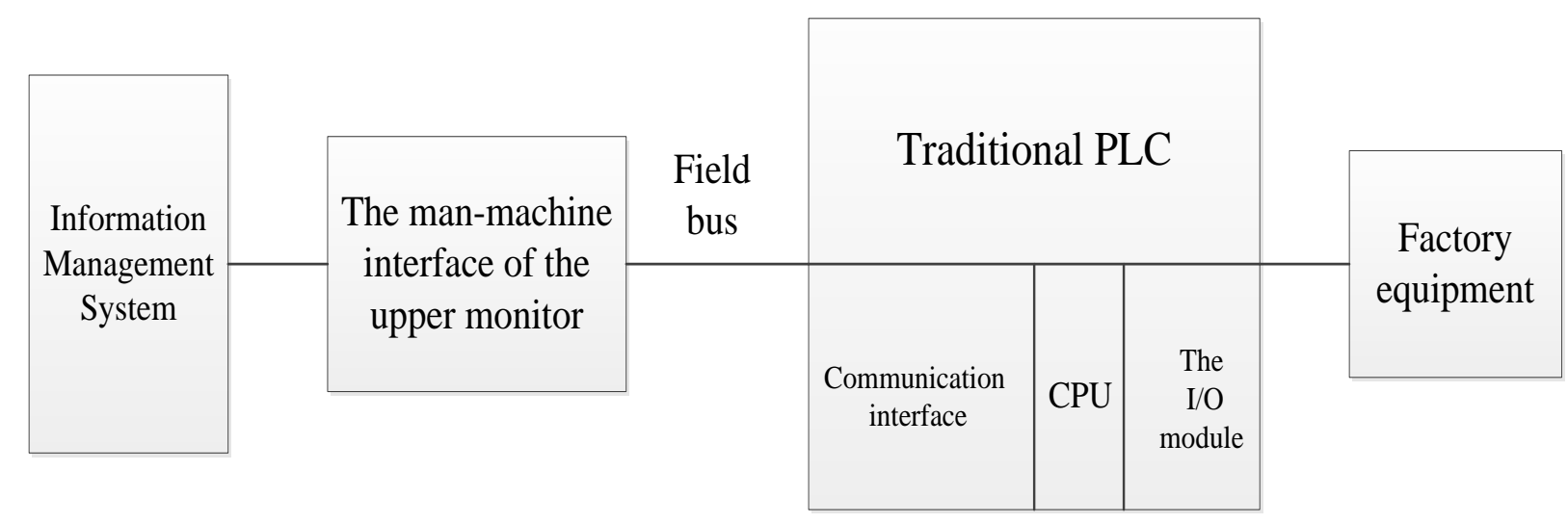

Figure1. The construction of traditional PLC

The construction of Soft PLC is shown as the figure 2.

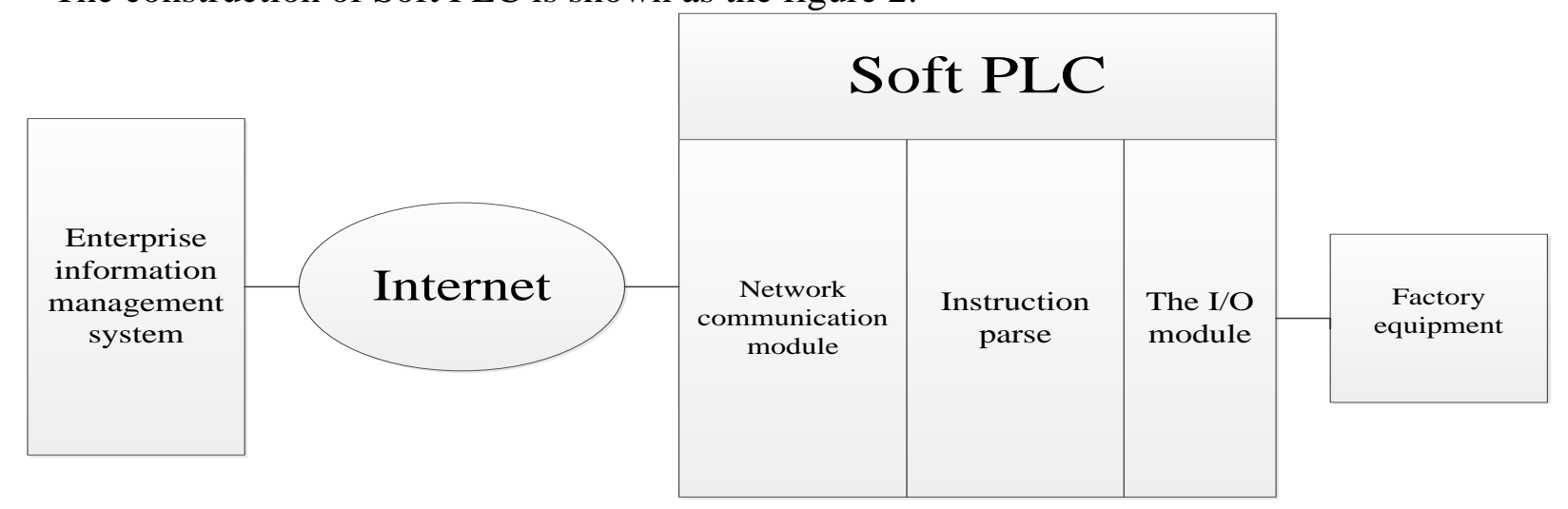

Figure2. The construction of Soft PLC

\section{The theory of soft PLC}

In general, the working process of soft PLC is similar to traditional one. First, users use PC soft PLC development system to develop a user program, and then compile and simulation, download to the soft PLC operating system after verification. The operating system will run the system, explain and then operate according to the traditional PLC format. Development system of PC could monitor every I / O status. In the process of running soft PLC, and it works in the same way as a traditional PLC, which incorporate cyclic scanning mode.

Soft PLC development system is a common integrated development environment that provides a standard IEC61131-3 programming language, grammar and the corresponding instructions, etc. for users to develop their own application in line with their own requirements. The operating system will perform the compiled application; meanwhile completing the service requested corresponding to the target system. For the I $\backslash \mathrm{O}$ subsystem, they will complete the on-site control signal input and output driven and managed in the operation of the system.

\section{The developmental trend of Soft PLC}

According to numerous professional organization and PLC manufacturers, they predict that the automation of PC-based will occupy $30 \%$ of the control system in 2010. In recent years, soft PLC has play an role in industrial area, and the usage is increasing every year. According to ARC surveys and estimation, the global market of soft PLC was 36 million US dollars in 1997 Soft PLC market reached \$ 145 million in 2000 and nearly double the in 2001. Nonetheless, due to the instability of the soft PLC, traditional PLC has been occupied for more than 30 years, the product is very mature, which lead to users are skeptical in using the soft PLC.

Due to the immaturity of the soft PLC product, in the coming few years, Soft PLC products will need to have the following improvement: 
I. Further enhance the openness of the current soft PLC products as its mainly based on Windows system, which is not supporting applications of other operating system; without the support of open hardware and software, it cannot guarantee the openness of the product; it does not allow users to change the underline control algorithms, it does not strictly comply with the international standards, etc.

II. Further enhancing the system reliability and timeliness, and this is the important factor for improving real-time of the operating system. Existing real-time operating system can only meet the basic need of industrial control. As some higher level real-time operating systems emerged, it will definitely and greatly enhance the timeliness and reliability of the soft PLC system.

\section{Design of the Soft PLC control system}

\section{System design plan}

In the control scheme, software platform can use either Windows NT, WincE or Linux operating system. Field bus cards, field bus and remote I / O modules (or general purpose I / O boards and I / O Terminal Board) is responsible for dealing with industrial control field, the gathered input signal will be processed by PLC operating system, and control application written by the soft PLC development system will also be interpreted and executed by the PLC operating system. The connection between the development system and the operating system is based on network communication channel, the output signals will be processed to a local (or remote control to complete appropriate local control (or long-range remote control and the control scheme is shown in Figure 3.

\section{Field bus}

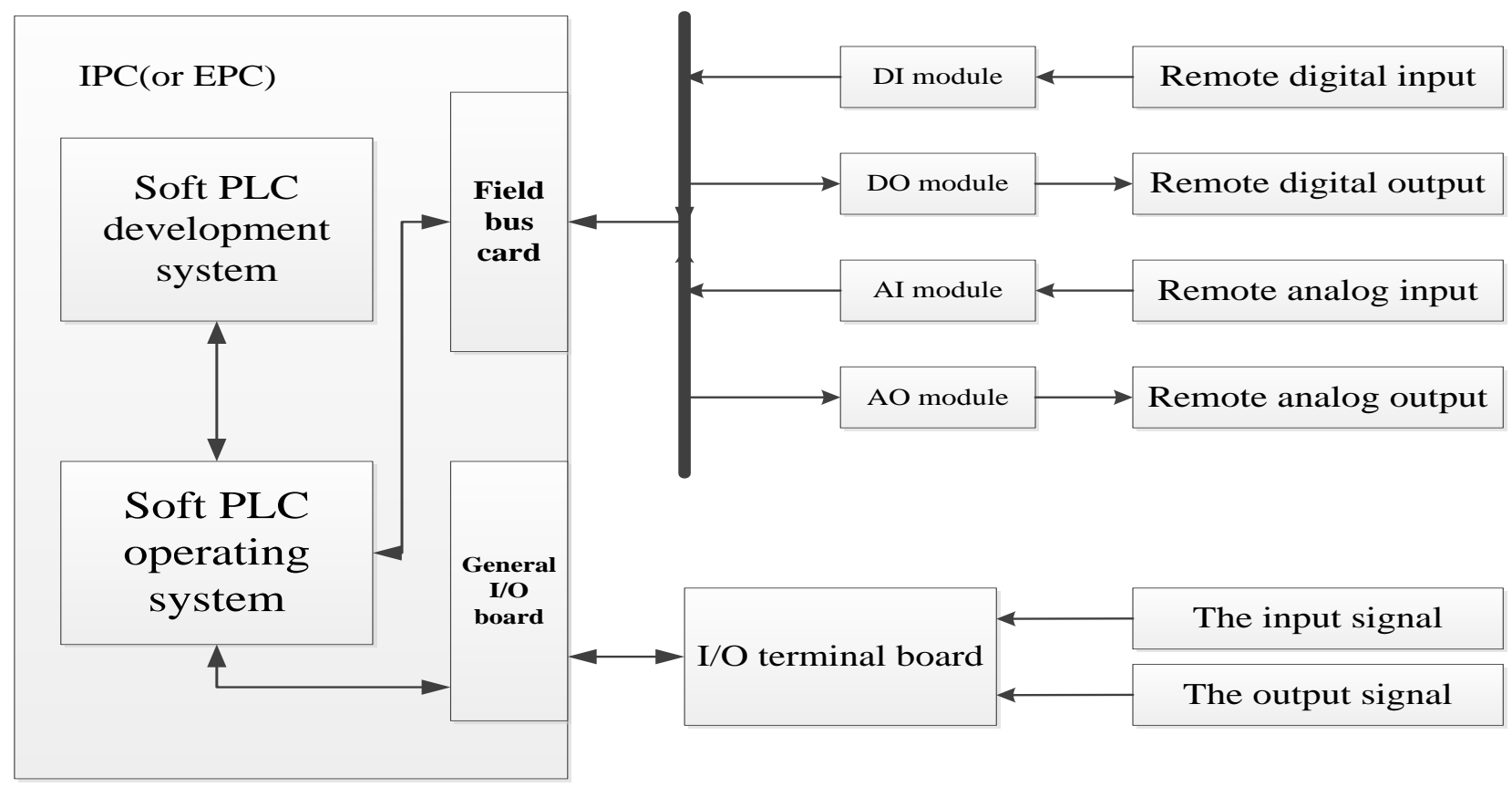

Figure3. System control scheme

In the design, function is defined as follows:

$$
\begin{array}{r}
r t n=G T_{-} \text {ExInpt }((\text { unsignedshort } *)((\text { unsignedchar } *) \\
\left.\left.- \text { memory }+_{-} \text {plc_mem_adress._in_plc }\right)\right) ; \\
r t n=G T_{-} \text {ExOpt }(8(\text { unsignedshort } *)((\text { unsignedchar } *) \\
\text {-memory } \left.\left.+_{-} \text {plc_mem_address._out_plc }\right)\right) ;
\end{array}
$$




\section{Soft PLC control system}

\section{System hardware}

Soft PLC system has good openness, various hardware platforms, including both traditional PLC hardware, and also having updated and embedded chip which is the excellent hardware platform for the network environment of PC or DCS system.

\section{System-specific module development}

Specific module will convert the PLC source program produced by universal program into operating system which can recognize the code, and downloaded to the operating system. The two main parties include: PLC source compiler, communication of development system and operating system.

A. Complier module implementation.Current system uses the design similar to PL/0 complier. Please refer to figure 4. In this compile mode, the process of converting instruction into target code can be divided into two stages: interpretation and generation. Instruction analysis is the process of comparing and accessing, which is making a comparison between the specific operation code, operand data and the data in the database, in order to determine correctness of the instruction. Forms Management application is used for establishing connections between reference variables, constants and the interpretation, the error handling application is used for providing error message for wrong location and the nature of error occurred during the process of instruction interpretation.

B. Communication between operating system and development process. In this system, Communication between operating system and development process has similar theory with general PC and traditional PLC. The host will first connect with PLC via cable for communication. Development system use RS232 / RS422 for connection between PLC and PC. The host and serial ports are RS232 and RS422 respectively; it requires a converter cable to connect the host and client for the purpose of conversion.

C. Communication is triggered by the host computer, the PC first sends the handshake, after PLC received it, and it sends back the signal to the handshake. The PC will start sending instructions after receiving back the signal; PLC will send data to the host after receiving the data. The host will check the data. if the data is wrong, it will request PLC resend it and displayed the process correctly.

\section{Conclusion}

Based on the analysis of soft PLC technology, its structure and principles, we propose to develop a PC-based soft PLC control scheme and also a suitable hardware platform development system. When users use the soft PLC operating system under the PC hardware platform, they can directly use this tool, which can save a lot of time and have important practical significance.

\section{Acknowledgements}

The key project of guangxi higher education teaching reform project (2013jgz181), Nanning vocational and technical college to build key course construction project between colleges (south post word [2012] no. 172) .

\section{References}

[1] Huang Y, LIN Y, YU H. Research and Implementation of the SoftPLC Technology [J][J]. Computer Engineering, 2004, 1: 063.

[2] Jianfeng Z F W X L, Wei W Y Z. An Approach to Soft PLC Editing System[J]. Computer Engineering and Applications, 2005, 7: 038.

[3] ZHU W, WANG W, DING H, et al. Open Architecture SoftPLC Based on Embedded PC [J][J]. 
Machinery \& Electronics, 2002, 3: 3-7.

[4] Kaiming Y H Y P Y, Jinsong W. Research and Development of RTLinux Based Soft PLC [J][J]. Computer Engineering and Applications, 2002, 22: 045.

[5] Jingang G, Jianchun C, Xiongwei L. Development of Soft-PLC of CNC System[J]. 2004. 